\title{
REVEALING A FULL QUANTUM LADDER BY NONLINEAR SPECTROSCOPY
}

\author{
D. Abramavičius \\ Institute of Chemical Physics, Faculty of Physics, Vilnius University, Sauletekio 9, 10222 Vilnius, Lithuania \\ Email: darius.abramavicius@ff.vu.lt
}

Received 16 June 2020; accepted 30 June 2020

\begin{abstract}
Coherent two-dimensional spectroscopy in the IR or the visible region is very effective for studying correlations, energy relaxation/transfer pathways in complex multi-chromophore or multi-mode systems. However, it is usually restricted up to two-quanta excitations and their properties. In this paper, an arbitrary level of excitation is suggested as the utility to scan nonlinear potential surfaces of quantum systems up to a desired excitation degree. This can be achieved by a simple three-pulse laser spectroscopy approach. Accurate evaluation of high-level anharmonicities as well as transition amplitudes can be directly obtained. Additionally, questions regarding the quantum nature of the probed system can be addressed by studying absolute peak positions.
\end{abstract}

Keywords: energy level structure, multiple-quanta excitations, pulsed laser spectroscopy

\section{Introduction}

Pulsed laser spectroscopy is one of the main material science utilities for revealing excited states dynamics. The forefront in the nonlinear domain, the two-dimensional coherent spectroscopy (2DIR in IR region, 2DES in visible region) [1, 2], has been applied in solid [3, 4], protein [5, 6], liquid [月, 8] as well as gaseous [9] materials. It has been also utilized in microscope configuration [10]. 2D spectroscopy evolved from the transient absorption spectroscopy. The two-pulse pump probe or transient absorption approach provides the time resolution by measuring the transient evolution of difference absorption induced by the excitation pump pulse and the detection probe pulse. Since the two pulses provide the time resolution as the delay between pump and probe pulses, the measurement essentially reflects the change of induced response of the sample in time. By adding a third laser pulse, e.g. in a three pulse photon echo peak shift (3PEPS) approach, the additional time delay of intraband coherence allows one to additionally follow the environmentinduced correlation functions of the energy gap fluctuations [11-13]. The pump probe and 3PEPS rely on the four-wave mixing ( $4 \mathrm{WM}$ ) process in the nonlinear system, however, from a theory point of view, the specific convolutions of the third-order molecular response function are performed and detected in these approaches [11, 14]. The convolutions are lifted by ultrashort excitation pulses imitating elementary perturbative excitations. A natural upgrade to the $4 \mathrm{WM}$ techniques then results in the four-pulse setup when the full deconvoluted information contained in the third-order response function is retrieved by the two-dimensional heterodyne detected coherent spectroscopy. Denoting the $i$ th pulse wavevector by $k_{i}$, in this case, the specific component of the four-wave mixing process can be associated with the system-field interaction configuration, and by collecting signals $-k_{1}+k_{2}+k_{3},+k_{1}-k_{2}+k_{3}, k_{1}+k_{2}-k_{3}$, all available 
information contained in the third-order response function with the largest possible time and frequency resolution can be gathered [1, 2, ].

Ideally, since each excitation pulse provides a single (one quantum) excitation with a dedicated wave vector, the specific peaks in spectra can be associated with a minimal set of interaction pathways. The obtained two-dimensional (2D) spectrum sometimes is denoted as representing a picture of 'quantum tomography' [15, 16 ].

In the visible optical regime, the $4 \mathrm{WM}$ measurements mainly demonstrate the properties of the single exciton manifold in $-k_{1}+k_{2}+k_{3}$ and $k_{1}-k_{2}+k_{3}$ configurations. The single excitation manifold is very important in physical chemistry as the single excitations are the main players in excitation transport, charge separation, charge transport processes in molecular and biological functional systems [17-24].

The double excitation manifold, i.e. the set of states, which are achieved by sequential two-photon absorption, participate in excited state absorption in transient absorption, 3PEPS and 2DES. The doubleexciton level structure or exciton-exciton correlation is directly achieved in the $k_{1}+k_{2}-k_{3}$ (double quantum coherence) detection [25, 26] or in the fifthorder 2D spectra where exciton-exciton annihilation becomes considerable [27-30].

While the dynamical aspects of energy migration are the main focus in electronic spectroscopy, the 2D spectroscopy approach in IR regime [31] has been utilized for molecular structure determination [2, 32, 33 and for tracking thermal fluctuations and chemical reaction dynamics [34-37] via vibrational resonances. Vibrational nonlinear spectroscopy allows direct measurement of diagonal and off-diagonal vibrational anharmonicities [38, 39]. These parameters are related to single and double quanta properties, hence, denote the lowest-order nonlinear properties of the complex vibrational potential surfaces [40-42]. Several spectroscopic methods, including infrared and ultraviolet absorption, Raman, jet-cooled laser-induced fluorescence, have been utilized to map out the whole series of vibrational quantum states of molecules in their ground and excited electronic states [43-45]. These multiple quanta properties are very important for numerous molecular processes such as chemical reactions [46, 47], isomerization or inversion [48-52], as well as energy transfer during inelastic collisions proceeding along vibrational pathways that are governed by vibrational potential energy surfaces [53, 54]. Development of spectroscopic approaches to reveal the whole vibrational (or electronic) potential surface is thus highly demanded and simple nonlinear spectroscopy probes may aid in this direction.

In this paper, a set of nonlinear optical measurements is suggested to directly measure multiplequanta up to a desired degree excitation properties. The obtained two-dimensional spectra then can be employed to reveal the energy level structure of a potential surface that is necessary for reconstruction of molecular potential surfaces. A side question regarding the quantum nature of vibrations can be addressed as well.

\section{Theory and simulations}

Optical excitations of a quantum system can be easily described by the response function theory at various orders with respect to the system-field interactions. Using a response function formalism the induced polarization's $j$ th-order to the field component is given by

$$
\begin{aligned}
& P^{(j)}(t)=\int_{0}^{\infty} \mathrm{d} t_{j} \ldots \int_{0}^{\infty} \mathrm{d} t_{1} S^{(j)}\left(t_{j} \ldots t_{1}\right) \times \\
& E\left(t-t_{j}\right) E\left(t-t_{j}-t_{j-1}\right) \ldots E\left(t-t_{j}-\ldots t_{1}\right)
\end{aligned}
$$

In an electric dipole approximation all even orders lead to vanishing response of isotropic samples. We next consider a single colour source excitation field with frequency resonant to one particular anharmonic molecular mode. Before hitting the sample, the field is split into a set of excitation fields

$$
\begin{aligned}
& E(t)=\sum_{i}^{N} E_{i}(t) \\
& =\sum_{i}^{N} A_{i}(t) \exp \left(\mathrm{ik}_{i} \mathbf{r}-\mathrm{i} \omega t\right)+\text { c.c. }
\end{aligned}
$$

Here $A_{i}(t)$ is a slowly varying amplitude of the $i$ th component whose wavevector is $\mathbf{k}_{i}$. In the nonlinear regime the phase matching phenomenon is an additional necessary companion in the optical spectroscopy. Indeed, multiplication of such fields in Eq. (1) results in superpositions of wavevectors, and thus the signals are being generated with the specific new signal wave vectors given by $\mathbf{k}_{s}=$ $n_{1} \mathbf{k}_{1}+n_{2} \mathbf{k}_{2}+\ldots n_{N} \mathbf{k}_{N^{*}}$. Here $N$ is the number of incoming fields, $n_{1}, \ldots, n_{N}$ are integers each taking values in the interval $-j,-j+1 \ldots j-1, j$, where $j$ is 
the order of nonlinearity, and we have additionally $\left|n_{1}\right|+\left|n_{2}\right|+\ldots+\left|n_{N}\right|=j$.

Spatial detuning of laser rays can be used to reveal specific output wavevectors. The collinear geometry can be also used to increase the field overlap region and specific phase matched configuration can be obtained by phase cycling. Phase cycling for $\mathrm{N}$ incoming pulses is formally equivalent to the spatial Fourier transformation in $N$ dimensions. To isolate an $m$ th order component ( $m+1$ wave mixing) on a single dimension one needs to use at least $m+1$ equally distributed phase values as dictated by periodicity of Fourier transform and Nyquist theorem (see Fig. 1(a)). Indeed, the discrete transform of equally spaced $M$ samples in the $x$ dimension of a function $f\left(x_{i}\right)=f_{i}$ yields $M$ samples in the conjugate $k_{x}$ dimension. So for distinguishing \pm 1 output values one needs to provide $M=4$ input distinct values corresponding to $-1 \Delta_{x^{\prime}}, 0 \Delta_{x^{\prime}}+1 \Delta_{x^{\prime}}, 2 \Delta_{x}$ or $1 k_{x}, 0 k_{x^{\prime}},+1 k_{x^{\prime}}$ $+2 k_{x}$ samples. Notice that in the $M=4$ case due to the periodicity of Fourier transform $-2 k_{x} \equiv+2 k_{x}$ cannot be distinguished.

At first, we may consider designing high-order multidimensional spectroscopy to probe highly lying excited states and reveal the complete nonlinear response function in the spirit of 2DES. Notice that at the 3rd order to the field by adding the third harmonic generation configuration, $k_{s}=k_{1}+k_{2}+k_{3}$, the number of independent phase matching components is $2^{3} / 2=4$ (half of $2^{3}$ components are (a)

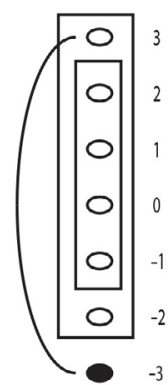

(b)

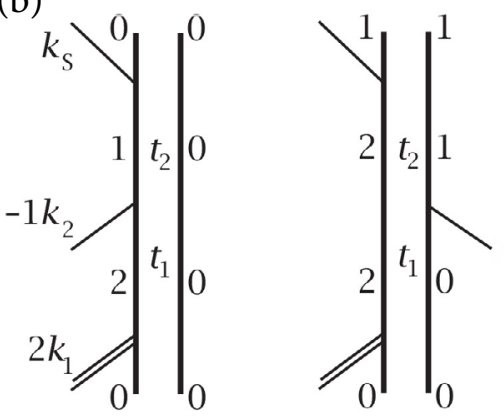

(c)

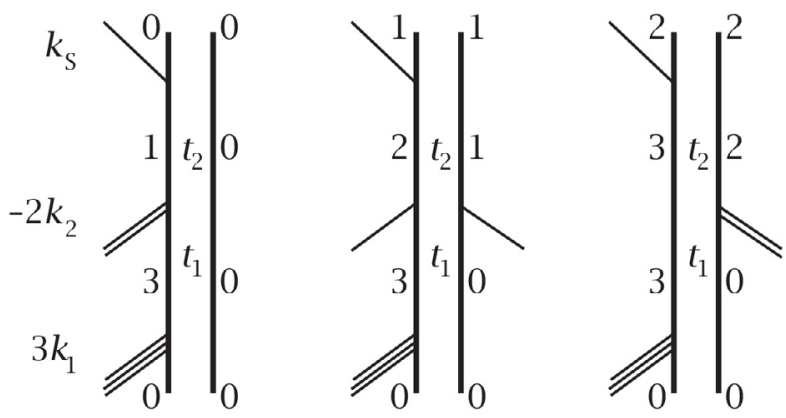

(d)
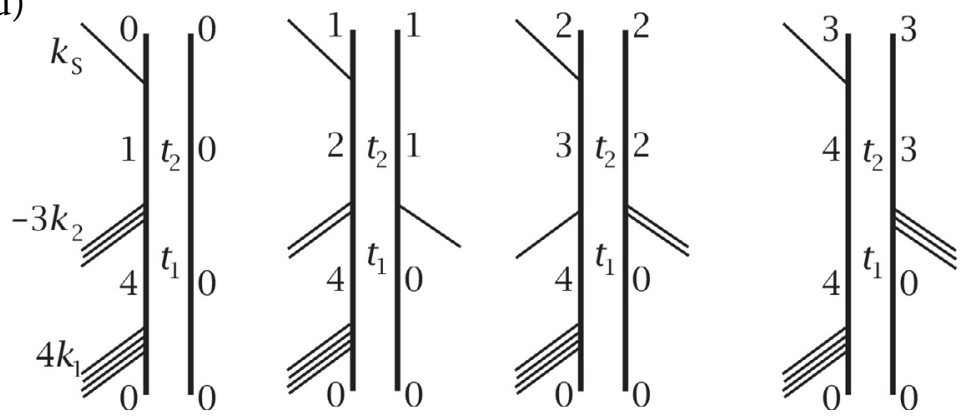

Fig. 1. (a) Resolution of wave vector components in the phase matching process as viewed from the spatial Fourier transform. Feynman diagrams for the $k_{s}=n k_{1}-(n-1) k_{2}$ phase matching configuration with two excitation pulses: (b) at 3 rd $(n=2)$, (c) at 5th $(n=3),(d)$ and at 7 th $(n=4)$ order to the field. 
conjugate to each other), at the $j$ th order we thus have $2^{j} / 2$. Hence, in order to gather the complete information available at $j$ th order, the number of phase matched configurations which have to be inspected scales exponentially with the order in the field.

Such 'complete-information' way of thinking calls for an experiment where for the $j$ th order we should consider $j$ laser pulses. However, it becomes impossible to continue in this spirit as the dimensionality of phase matching configurations and the dimensionality of signal as a function of time delays grows up and the amount of gathered data becomes prohibitively large.

Hence, as higher-energy and quanta states become populated in higher-order spectroscopies, the focus of the problems must shift towards the multiple-excitation characteristics. The most important information then, for example, is the energy level configuration or, in other words, the shape of potential surface [55]. It should be noted that this used to be the main focus of 2DIR where vibrational anharmonicities have been resolved [31].

Notice that the high-order nonlinear interaction process is not necessarily related to a large number of laser pulses. Indeed, a single field induces response at an arbitrary order to the field and the phase matching unravels specific interaction pathways. The ideal approach is to use the smallest number of excitation pulses to reveal the desired information on $n$-quanta energy levels. A sequential process may be designed by two pulses to drive the excitation up and down the ladder to the quantum states and then detect the optical coherence field.

Consider, for example, a response induced by two incoming laser pulses. The first pulse corresponds to wavevector $k_{1}$ while the second to $k_{2}$. Let us inspect the induced polarization detected at $k_{s}=n k_{1}-(n-1) k_{2}$, where $n$ is an arbitrary integer. The Feynman diagrams corresponding to such process are given in Fig. 1 (b-d). In this setup, during the first delay interval $t$ the state of the system is given by coherent superposition of the ground state and the $n$th excited state that is reached by absorption of $n$ photons. In the case of small anharmonicity, the corresponding density matrix element oscillates with the frequency approximately equal to $n \omega_{0}$, where $\omega_{0}$ is the frequency of the photon, provided anharmonicity is small. The following $n-1$ interactions (either on the left or on the right of the diagram) with the second pulse after delay $t_{1}$ generate interband coherences that range from $|1\rangle\langle 0|$ to $|n\rangle\langle n-1|$. The diagram thus projects information about $1,2, \ldots, n$ quanta excited states onto a two-dimensional function of delays $t_{1}$ and $t_{2}$. The latter can be Fourier transformed into a twodimensional spectrum $S\left(\omega_{1}, \omega_{2}\right)$.

Notice that considering $n$ quanta excitations the strongest transitions are between $n \rightarrow n \pm 1$ levels; these transitions originate from a single light quantum absorption/emission. Hence, in the $k_{s}=n k_{1}-(n-1) k_{2}$ configuration all involved transitions are of single quantum absorption/ emission, so the signal intensity is never scaled by a small anharmonic correction of the transition dipole.

As an example, the multiple quanta spectra of a Morse oscillator are presented in the following. The Morse oscillator is a unique anharmonic quantum system with its energy levels and transition amplitudes available analytically [56]. The potential surface of the oscillator can be given by

$$
V(x)=D(1-\exp (-\alpha x))^{2}
$$

with $D$ being the classical ionization energy, where $\alpha$ is the curvature parameter of the potential valley. For the case when mass of the oscillator is $1 / 2$, the oscillator fundamental frequency is equal to $\omega_{\alpha}=2 \alpha \sqrt{D}$. By taking for convenience $\alpha=1$ we find that $D$ is the only anharmonicity parameter. Denoting $D=(\mathcal{N}+1 / 2)^{2}$, the integer part of $\mathcal{N}+1 / 2$ (which we denote by $N=\operatorname{Int}[\mathcal{N}+1 / 2]$ ) equals the number of discrete quantum levels. Essentially, the system is more anharmonic as the number of discrete energy levels gets smaller and the oscillator turns into harmonic system with $D \rightarrow \infty$. Energy spectrum of the discrete states of the oscillator in this case is

$$
E_{n}=-(\mathcal{N}-n)^{2}
$$

with $n=0,1 \ldots N-1$. In the following we assume that the dissociation is not relevant and we do not consider the continuum part of the spectrum, since its contribution to the spectrum is quite week (checked numerically, not shown).

The next required quantity is the optical transition dipole moment. In order to determine it, it is 
necessary to define the polarization operator. The polarization operator for a harmonic oscillator is often assumed to be proportional to the coordinate operator, $\hat{P}=\mu \hat{\mathrm{x}}$. We next assume that the optical field only involves transitions between adjacent energy levels and other transitions are off-resonant. We thus consider only matrix elements $\langle n|\hat{P}| n+1\rangle$ [56]

$$
\begin{aligned}
& \mu_{n} \equiv \mu\langle n|\hat{x}| n+1\rangle= \\
& \mu \frac{\sqrt{(\mathcal{N}-n)(\mathcal{N}-n-1)}}{\mathcal{N}-n-1 / 2}\left[\frac{n+1}{2 N-n}\right]^{\frac{1}{2}},
\end{aligned}
$$

which for large $\mathcal{N} \gg n$ reduces to the result of a harmonic oscillator, $\mu_{n+1} / \mu_{n} \approx \sqrt{(n+2) /(n+1)}$.

Consequently, we can write analytically the resulting expressions for the $2 \mathrm{D}$ peak intensities. For example, for $k_{s}=4 k_{1}-3 k_{2}$ we have the set of peaks with their amplitudes in the harmonic case approximately equal to $4 ! \mu_{0}$ since the system in each diagram absorbs and emits up to 4 quanta. In general, for the signal component $k_{s}=n k_{1}-(n-1) k_{2}$ the peak intensities will be $n ! \mu_{0}$.
All distinct diagrams have positions determined by resonant transition energies. The fundamental energy gap

$$
\omega_{0}=E_{1}-E_{0}=2 \mathcal{N}-1,
$$

and correspondingly the sequence of energy gaps

$$
E_{n+1}-E_{n}=\omega_{0}-2 n
$$

are linear with $n$. This sequence is directly observed in the $k_{s}=n k_{1}-(n-1) k_{2}$ configuration.

By using the diagram approach we can easily show what peaks will be visible in the $2 \mathrm{D}$ spectrum for various wavevector configurations for the Morse oscillator. The oscillation frequency in the $t_{1}$ interval is

$$
E_{n}-E_{0}=n \omega_{0}-\Delta_{n}
$$

with $\Delta_{n}=n(n-1)$, so the sets of peaks in the $k_{s}=n k_{1}-(n-1) k_{2}$ measurement will appear at frequency $w_{1}=n \omega_{0}-\Delta_{n}$. During $t_{2}$ the system will oscillate with frequencies corresponding to energy gaps $E_{i+1}-E_{i}=\omega_{0}-\Delta_{2} i$ for $i=0,1 \ldots n$. Such situation is demonstrated on the top of Fig. 2 .

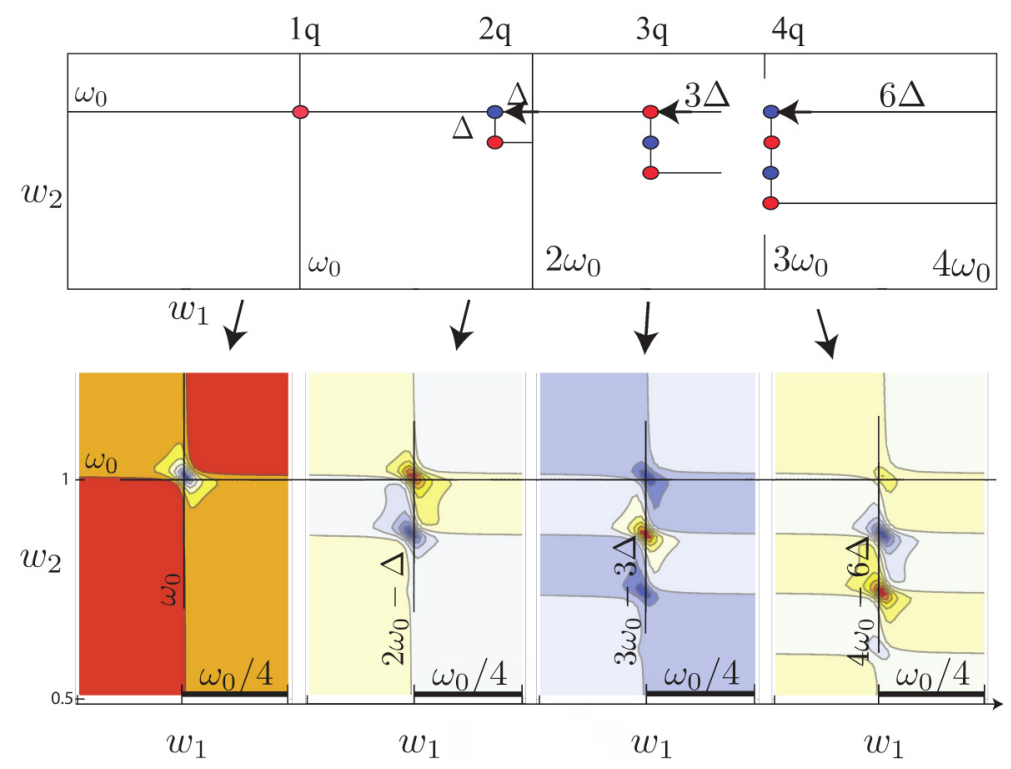

Fig. 2. Top: scheme of peaks in the $k_{s}=n k_{1}-(n-1) k_{2}$ measurement of the Morse oscillator corresponding to (from left to right) $n=1, n=2, n=3$ and $n=4 . \omega_{0}$ is the fundamental frequency and $\Delta$ is the lowest anharmonicity. Bottom: the spectrum of the Morse oscillator obtained from solving the Liouville equation and the signal (Eq. (11)) is reconstructed by phase cycling. The damping is included as an additional factor to the signal as $\exp \left(-\gamma\left(t_{1}+t_{2}\right)\right)$ with $\gamma=0.02 \omega_{0}$ so that all resonances are explicitly expressed. 
To demonstrate the above described approach in a more realistic scenario, we next present numerical simulations of the excitation and detection process of the Morse oscillator with dephasing and lineshapes. We set the oscillator parameter $\mathcal{N}=8$, so that the number of bound states is $N=8$. The initial state of the system is equal to the ground state $|0\rangle$. For the excitation process, which we represent as action by nonlinear excitation operator, $X_{\phi} \boldsymbol{\rho}_{0}$, we assume excitation by an optical field resonant to the fundamental frequency and with the bandwidth covering all transitions $n \rightarrow n+1$. In order to realize that the excitation/deexcitation is nonperturbative (as in the real experiment), we numerically integrate the interaction picture Schrödinger equation with the excitation field turned on. For the Liouville equation this results in the iteration of the equation

$$
\dot{\boldsymbol{\rho}}=-\mathrm{i}\left(\boldsymbol{\mu}_{\phi} \boldsymbol{\rho}-\boldsymbol{\rho} \boldsymbol{\mu}_{\phi}\right),
$$

where

$$
\boldsymbol{\mu}_{\phi}=A\left(\begin{array}{ccccc}
0 & \mu_{1} \mathrm{e}^{-\mathrm{i} \phi} & 0 & \ldots & 0 \\
\mu_{1} \mathrm{e}^{-\mathrm{i} \phi} & 0 & \mu_{2} \mathrm{e}^{-\mathrm{i} \phi} & \ldots & 0 \\
0 & \mu_{2} \mathrm{e}^{-\mathrm{i} \phi} & 0 & \ldots & 0 \\
\ldots & \ldots & \ldots & \ldots & \ldots \\
0 & 0 & 0 & \ldots & 0
\end{array}\right)
$$

is the transition dipole operator with only RWA terms including the excitation intensity $A$ and phase $\phi$ (the phase is necessary for phase cycling to recover correct phase matching contributions). This equation is propagated for an infinitesimal time interval $\delta t \rightarrow 0$ with the given intensity $A$, so that $A \delta t$ is not vanishing and can be tuned to populate the chosen number of states.

So the protocol is as follows. We take the initial density matrix

$$
\boldsymbol{\rho}_{0}=\left(\begin{array}{rrr}
1 & 0 & \ldots \\
0 & 0 & \ldots \\
\ldots & \ldots & \ldots
\end{array}\right),
$$

perform excitation $X_{\phi_{1}} \boldsymbol{\rho}_{0}$ with phase $\phi_{1}$, then we perform the density matrix propagation according to the Liouville equation with the field switched off to obtain $\mathcal{G}\left(t_{1}\right) X_{\phi_{1}} \boldsymbol{\rho}_{0}$. The second excitation follows with the phase $\phi_{2}: X_{\phi 2} \mathcal{G}\left(t_{1}\right) X_{\phi_{1}} \boldsymbol{\rho}_{0}$, propagation with the field off for time delay $t_{2}: \mathcal{G}\left(t_{2}\right) X_{\phi_{1}} \mathcal{G}\left(t_{1}\right) X_{\phi_{1}} \boldsymbol{\rho}_{0}$, and finally we perform the final detection to obtain the signal

$$
S\left(t_{1}, t_{2}\right)=\operatorname{Tr}\left\{\boldsymbol{\mu}_{L} \cdot \mathcal{G}\left(t_{2}\right) X_{\phi_{2}} \mathcal{G}\left(t_{1}\right) X_{\phi_{1}} \boldsymbol{\rho}_{0}\right\} .
$$

We emphasize the difference between this expression and standard response function theory, where each excitation event is a linear operation. In our approach, we assume that each operation $X_{\phi_{1}} \rho$ is nonlinear and thus generates the whole spectrum of harmonics.

On the bottom of Fig. 2 we present a series of 2D spectra after the Fourier transform $S\left(t_{1}, t_{2}\right) \rightarrow$ $\mathrm{S}\left(\omega_{1}, \omega_{2}\right)$ for the signal $k_{S}=n k_{1}-(n-1) k_{2}$ with $n=1$, $2,3,4$ that correspond to the nonlinear signals at 1 , $3,5,7$ order. The line broadening was kept small.

The common critique to these types of simulations is that the line broadening has to be maintained small in order to observe the peak pattern and to determine the anharmonicity. However, this type of signal is not an issue because we observe not a single pair of peaks, but the whole train of peaks. The same simulations of $2 \mathrm{D}$ spectra with large line broadening $\left(\gamma=0.1 \omega_{0}\right)$ is shown in Fig. 3. On the top row, we can observe that overlapping peaks become squeezed, but the number of peaks and peak splitting can be uniquely determined.

Additional question on the quantum nature of oscillations can be addressed in this case. We additionally show the corresponding classical simulation results, i.e. for the same type of Morse potential but obtained from the purely classical equations of motion. We find that the peak pattern is quite similar, however, its overall absolute position becomes shifted; the series of peaks becomes positioned symmetrically with respect to the theoretical central point equal to $\left(w_{2}, w_{1}\right)=\left(\omega_{0}, n \omega_{0}\right)$, while the series is shifted in the case of a quantum oscillator.

\section{Discussion and conclusions}

So where is the next level of pulsed laser spectroscopy? In this paper, the direction towards higherorder nonlinear interactions is considered. The fifthorder coherent spectroscopy has been considered in the IR and the visible region and the complex information on the correlations in quantum systems has been represented in 3DIR [57], 3DES [58-60] or $3 \mathrm{D}$ fluorescence [61]. By spreading frequencies into the third spectral dimension high-frequency 

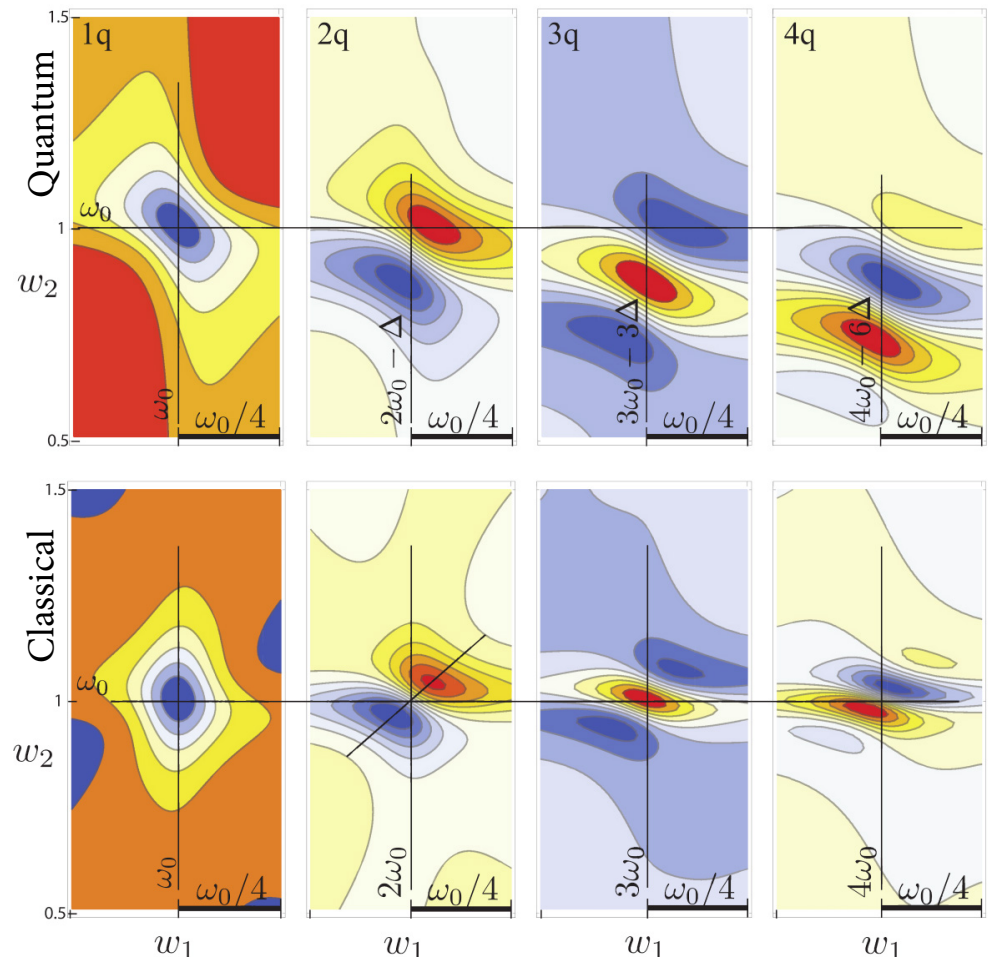

Fig. 3. Numerical simulations of the $k_{s}=n k_{1}-(n-1) k_{2}$ signals of the Morse oscillator corresponding to (from left to right) $n=1$, $n=2, n=3$ and $n=4$ cases for the quantum (top) and classical (bottom) oscillators. $\omega_{0}$ is the fundamental frequency and $\Delta$ is the lowest anharmonicity. Line broadening parameter is set to $\gamma=0.1 \omega_{0}$.

vibronic modes [58], double-exciton resonances [27-29] or specific exciton relaxation pathways [59. 60] have been revealed. To collect the complete information, five excitation pulses are needed for the generation of an interaction pathway-specific detailed picture. The full set of information then comes up in five dimensions as five independent time intervals between interactions should be considered. Utilizing, e.g. the gradient-assisted photon echo (GRAPE) methodology, the fifth-order nonlinear polarization through three sequential single-quantum coherences has been measured in parallel [58] and presumably could be extended for the 5D measurement. However, the information contents in the corresponding full-scale experiments become huge, and interpretation becomes challenging.

The approach presented in this paper is not related to extracting all possible information at a certain order, but, instead, is specifically geared to extract only a small amount of information, but with a minimal amount of effort with high accuracy. A similar approach has been utilized to gain highorder electronic correlations in quantum wells [62].
This specificity can be demonstrated with respect to the vibrational anharmonicity that is often targeted in 2DIR of proteins. Simple numerical simulations in Fig. 4 demonstrate that the anharmonicity that is being extracted from a simple 2DIR measurement may be distorted by large broadening, and thus, cannot be used to accurately determine the corresponding Hamiltonian parameters. Meanwhile, Fig. 3(top) demonstrates that the anharmonicity is revealed not only on vertical but also on horizontal axes and can be accessed without interference from line overlaps.

It should be noted that other anharmonic properties should be included when using more accurate simulation approaches. For example, $0-2,0-3$, etc. transition dipole elements of the Morse potential are not zero, additionally, at high excitation levels, continuum states can be reached. On the spectroscopy side, this could indicate an interesting turn for the spectroscopy analysis, while, on the chemical side, this can be used for initial chemical reactions or molecular isomerization.

The approach described in this paper, in general, applies not only to vibrational excitations but also to 


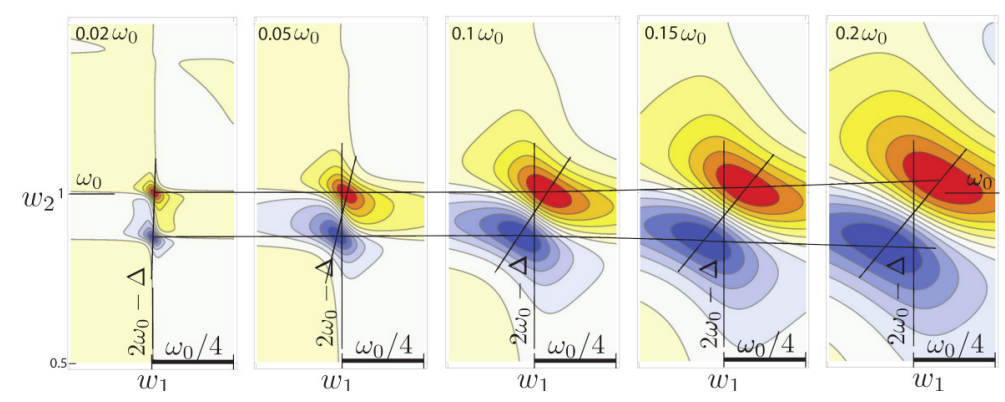

Fig. 4. Numerical simulations of a simple 2DIR signal of the Morse oscillator with the increasing linewidth parameter $\gamma$ shown in the insets of the subplots.

electronic states of molecules or atoms, but would require careful analysis of the spectra as electronic oscillators are highly anharmonic and positions of double-triple quanta excitations are poorly a priori anticipated (that may not be the case for Rydberg atoms in gas phase, etc.).

The other question that comes up is what kind of new information can be extracted from such multi-dimensional signals. In electronic time resolved spectroscopy the excitation relaxation pathways are primary targets and, for example, according to the perturbation theory the relaxation process depends on the two-point fluctuation correlation function [63]. In principle, the $n$ thorder nonlinear signal is dependent on the $n+1$ point correlation function [14]. So the first-order response (linear absorption) is related to the twopoint correlation function $C\left(t_{2}, t_{1}\right)$ which reduces to $C\left(t_{2}-t_{1}\right)$ due to the assumption of ergodicity (equilibrium before the measurement), and is in principle sufficient to reveal the relaxation phenomena. Indeed, the dephasing and relaxation processes determined spectral lineshape positions and broadening. The correlation function here is considered in a broad sense to include also the electronic degrees of freedom. The third-order response function maps the four-point correlation functions. The electronic degrees of freedom (i.e. energy level structure) become reflected onto the $2 \mathrm{D}$ plot in two-dimensional coherent spectroscopy, while the vibrational part of the correlation function results in spectral lineshapes. However, assuming the fluctuation process as a Gaussian process, all multi-point correlation functions split into a set of simple two-point, $C\left(t_{i}-t_{j}\right)$, correlation functions. Consequently, going to higher orders does not necessarily generate novel information. Recently, at the fifth order to the field the exciton-exciton annihilation processes have been considered that encapsulate the novel four-particle correlation properties as they originate from $\hat{a}^{\dagger} \hat{a}^{\dagger} \hat{a} \hat{a}$ terms [27-29]; however, focusing on exciton migration again restricts the focus only to the one-particle diffusion problem.

The level of complexity becomes overwhelming in biological systems where interactions between electronic and vibrational degrees of freedom may have important implications for rapid and efficient energy transfer. Development of higher-order spectroscopies that may enable unambiguous assignment of signals to specific pathways is very active. Highly nonlinear Raman approaches have been proposed since 2000 [64]. 2D resonance Raman techniques denoted by femtosecond stimulated Raman spectroscopy (FSRS) yield a subset of the quantum pathways [65-67], reveal electronic and vibrational interactions, however, suffer from contributions from cascades of third-order processes [68-70]. A direct correlation between pulse driven low-frequency modes such as phonons, vibrations and (multi-)excitons with quantum coherence selectivity through control of resonance can specifically be probed by the fifth-order gradientassisted multidimensional electronic Raman spectroscopy (GAMERS) free from low-order cascades [71]. Hence, target-specific approaches are advantageous.

It should be noted that large field intensities are often necessary to achieve a good signal to noise ratio at high nonlinearity orders. Then high-order nonlinearities become involved in apparent low-degree phase matching configurations that are supposed to be sensitive only to low orders to the field. For example, the traditional photon echo 
$k_{s}=-k_{1}+k_{2}+k_{3}$ configuration may be plagued by other superpositions, like $k_{s}=-k_{1}-k_{1}+k_{1}+k_{2}+k_{3}$, etc., which come from the fifth order [27, 29]. Similarly, seventh, ninth, etc. orders contribute to the specific phase matching configuration. Therefore, studying high-order nonlinearities in seemingly low-order phase matching configurations, e.g. exciton annihilation in pump-probe, carries a large nonzero background. Instead, in our suggested approach we have a zero background signal that allows one to cleanly identify the specific order without 'poisoning' by higher orders when the lowest possible excitation intensity is maintained. Alternatively, low-order cascading signals may obscure the highly nonlinear signals in simple phase matching configurations. The approach suggested in this paper could be free from cascading contributions if spatial phase matching in non-collinear laser configuration is applied. In the collinear scheme with phase matching the error control must be very rigorous to avoid cascading.

Concluding, a simple two-exitation-pulse nonlinear spectroscopy approach is proposed to reveal the quantum ladder of states in an anharmonic oscillator. Consequently, this could be employed in reconstruction of the whole potential surface at a desired excitation level.

\section{Acknowledgements}

The author acknowledges financial support of the Research Council of Lithuania (Grant No. SMIP20-47)

\section{References}

[1] D. Abramavicius, B. Palmieri, D.V. Voronine, F. Šanda, and S. Mukamel, Coherent multidimensional optical spectroscopy of excitons in molecular aggregates; quasiparticle versus supermolecule perspectives, Chem. Rev. 109, 2350-2408 (2009), https://doi.org/10.1021/cr800268n

[2] W.Zhuang, T. Hayashi, and S. Mukamel, Coherent multidimensional vibrational spectroscopy of biomolecules: Concepts, simulations, and challenges, Angew. Chemie Int. Ed. 48, 3750-3781 (2009), https://doi.org/10.1002/anie.200802644

[3] M. Cho, Two-Dimensional Optical Spectroscopy (CRC Press, 2009).
[4] P. Wen, G. Christmann, J.J. Baumberg, and K.A. Nelson, Influence of multi-exciton correlations on nonlinear polariton dynamics in semiconductor microcavities, New J. Phys. 15, 025005 (2013), https://doi.org/10.1088/13672630/15/2/025005

[5] G.S.Schlau-Cohen, A. Ishizaki, and G.R. Fleming, Two-dimensional electronic spectroscopy and photosynthesis: Fundamentals and applications to photosynthetic light-harvesting, Chem. Phys. 386, 1-22 (2011), https://doi.org/10.1016/j. chemphys.2011.04.025

[6] M. Reppert and A. Tokmakoff, Computational amide I 2D IR spectroscopy as a probe of protein structure and dynamics, Annu. Rev. Phys. Chem. 67, 359-386 (2016), https://doi.org/10.1146/annurev-physchem-040215-112055

[7] S. Hume, G. Hithell, G.M. Greetham, P.M. Donaldson, M. Towrie, A.W. Parker, M.J. Baker, and N.T. Hunt, Measuring proteins in $\mathrm{H}_{2} \mathrm{O}$ with 2D-IR spectroscopy, Chem. Sci. 10, 6448-6456 (2019), https://doi.org/10.1039/c9sc01590t

[8] M. Thämer, L. De Marco, K. Ramasesha, A. Mandal, and A. Tokmakoff, Ultrafast 2D IR spectroscopy of the excess proton in liquid water, Science 350, 78-82 (2015), https://doi.org/10.1126/science.aab3908

[9] L. Bruder, U. Bangert, M. Binz, D. Uhl, R. Vexiau, N. Bouloufa-Maafa, O. Dulieu, and F. Stienkemeier, Coherent multidimensional spectroscopy of dilute gas-phase nanosystems, Nat. Commun. 9, 4823 (2018), https://arxiv.org/abs/1806.08314, https://doi.org/10.1038/s41467-018-07292-w

[10]V. Tiwari, Y. Acosta Matutes, A.T. Gardiner, T.L.C. Jansen, R.J. Cogdell, and J.P. Ogilvie, Spatially-resolved fluorescence-detected two-dimensional electronic spectroscopy probes varying excitonic structure in photosynthetic bacteria, Nat. Commun. 9, 4219 (2018), https://arxiv. org/abs/1802.04395, https://doi.org/10.1038/ s41467-018-06619-x

[11]W.P. De Boeij, M.S. Pshenichnikov, and D.A. Wiersma, System-bath correlation function probed by conventional and time-gated stimulated photon echo, J. Phys. Chem. 100, 11806-11823 (1996), https://doi.org/10.1021/jp961039m 
[12]K.F. Everitt, E. Geva, and J.L. Skinner, Determining the solvation correlation function from three-pulse photon echoes in liquids, J. Chem. Phys. 114, 1326-1335 (2001), https://doi. org/10.1063/1.1332811

[13]E.A. Gibson, Z. Shen, and R. Jimenez, Threepulse photon echo peak shift spectroscopy as a probe of flexibility and conformational heterogeneity in protein folding, Chem. Phys. Lett. 473, 330-335 (2009), https://doi.org/10.1016/j. cplett.2009.04.002

[14]S. Mukamel, Principles of Nonlinear Optical Spectroscopy (Oxford University Press, New York, 1995).

[15]J. Yuen-Zhou and A. Aspuru-Guzik, Quantum process tomography of excitonic dimers from two-dimensional electronic spectroscopy. I. General theory and application to homodimers, J. Chem. Phys. 134, 134505 (2011), https://doi. org/10.1063/1.3569694

[16]D. Abramavicius, V. Butkus, J. Bujokas, and L. Valkunas, Manipulation of two-dimensional spectra of excitonically coupled molecules by narrow-bandwidth laser pulses, Chem. Phys. 372, 22-32 (2010), https://doi.org/10.1016/j. chemphys.2010.04.015

[17]E. Collini, C.Y. Wong, K.E. Wilk, P.M.G. Curmi, P. Brumer, and G.D. Scholes, Coherently wired light-harvesting in photosynthetic marine algae at ambient temperature, Nature 463, 644-647 (2010), https://doi.org/10.1038/nature08811

[18]E. Meneghin, A. Volpato, L. Cupellini, L. Bolzonello, S. Jurinovich, V. Mascoli, D. Carbonera, B. Mennucci, and E. Collini, Coherence in carotenoid-to-chlorophyll energy transfer, Nat. Commun. 9, 3160 (2018), https://doi. org/10.1038/s41467-018-05596-5

[19]D. Paleček, P. Edlund, S. Westenhoff, and D. Zigmantas, Quantum coherence as a witness of vibronically hot energy transfer in bacterial reaction center, Sci. Adv. 3, e1603141 (2017), https://doi.org/10.1126/sciadv.1603141

[20] G.D. Scholes, G.R. Fleming, L.X. Chen, A. AspuruGuzik, A. Buchleitner, D.F. Coker, G.S. Engel, R. van Grondelle, A. Ishizaki, D.M. Jonas, et al., Using coherence to enhance function in chemi- cal and biophysical systems, Nature 543, 647-656 (2017), https://doi.org/10.1038/nature21425

[21]J. Pan, A. Gelzinis, V. Chorošajev, M. Vengris, S.S. Senlik, J.-R. Shen, L. Valkunas, D. Abramavicius, and J.P. Ogilvie, Ultrafast energy transfer within the photosystem II core complex, Phys. Chem. Chem. Phys. 19(23), 15356-15367 (2017), https://doi.org/10.1039/C7CP01673E

[22]F.D. Fuller, J. Pan, A. Gelzinis, V. Butkus, S.S. Senlik, D.E. Wilcox, C.F. Yocum, L. Valkunas, D. Abramavicius, and J.P. Ogilvie, Vibronic coherence in oxygenic photosynthesis, Nat. Chem. 6, 706-711(2014), https://doi.org/10.1038 nchem.2005

[23]J. Lim, D. Paleček, F. Caycedo-Soler, C.N. Lincoln, J. Prior, H. von Berlepsch, S.F. Huelga, M.B. Plenio, D. Zigmantas, and J. Hauer, Vibronic origin of long-lived coherence in an artificial molecular light harvester, Nat. Commun. 6, 7755 (2015), https://doi.org/10.1038/ncomms8755

[24]E. Thyrhaug, R. Tempelaar, M.J.P. Alcocer, K. Žídek, D. Bína, J. Knoester, T.L.C. Jansen, and D. Zigmantas, Identification and characterization of diverse coherences in the Fenna-MatthewsOlson complex, Nat. Chem. 10, 780-786 (2018), https://doi.org/10.1038/s41557-018-0060-5

[25]D. Abramavicius, A. Nemeth, F. Milota, J.Sperling, S. Mukamel, and H.F. Kauffmann, Weak exciton scattering in molecular nanotubes revealed by double-quantum two-dimensional electronic spectroscopy, Phys. Rev. Lett. 108, 067401 (2012), https://doi.org/10.1103/PhysRevLett.108.067401

[26]J. Kim, S. Mukamel, and G.D. Scholes, Twodimensional electronic double-quantum coherence spectroscopy, Acc. Chem. Res. 42, 13751384 (2009), https://doi.org/10.1021/ar9000795

[27]J. Dostál, F. Fennel, F. Koch, S. Herbst, F. Würthner, and T. Brixner, Direct observation of exciton-exciton interactions, Nat. Commun. 9 , 2466 (2018), https://doi.org/10.1038/s41467-01804884-4

[28]B. Brüggemann and T. Pullerits, Nonperturbative modeling of fifth-order coherent multidimensional spectroscopy in light harvesting antennas, New J. Phys. 13, 025024 (2011), https://doi. org/10.1088/1367-2630/13/2/025024 
[29]V. Bubilaitis, J. Hauer, and D. Abramavicius, Simulations of pump probe spectra of a molecular complex at high excitation intensity, Chem. Phys. 527, 110458 (2019), https://doi.org/10.1016/j. chemphys.2019.110458

[30]J. Süß, J. Wehner, J. Dostál, T. Brixner, and V. Engel, Mapping of exciton-exciton annihilation in a molecular dimer via fifth-order femtosecond twodimensional spectroscopy, J. Chem. Phys. 150, 104304 (2019), https://doi.org/10.1063/1.5086151

[31]M.D. Fayer (ed.), Ultrafast Infrared Vibrational Spectroscopy (CRC Press, 2013).

[32]A. Remorino and R.M. Hochstrasser, Threedimensional structures by two-dimensional vibrational spectroscopy, Acc. Chem. Res. 45, 18961905 (2012), https://doi.org/10.1021/ar3000025

[33]A.W. Smith, J. Lessing, Z. Ganim, C.S. Peng, A. Tokmakoff, S. Roy, T.L.C. Jansen, and J. Knoester, Melting of $\beta$-hairpin peptide using isotope-edited 2D IR spectroscopy and simulations, J. Phys. Chem. B 114, 10913-10924 (2010), https://doi.org/10.1021/jp104017h

[34]J.M. Anna, C.R. Baiz, M.R. Ross, R. McCanne, and K.J. Kubarych, Ultrafast equilibrium and non-equilibrium chemical reaction dynamics probed with multidimensional infrared spectroscopy, Int. Rev. Phys. Chem. 31, 367-419 (2012), https://doi.org/10.1080/0144235X.2012.716610

[35]M.D. Fayer, D.E. Moilanen, D. Wong, D.E. Rosenfeld, E.E. Fenn, and S. Park, Water dynamics in salt solutions studied with ultrafast twodimensional infrared (2D IR) vibrational echo spectroscopy, Acc. Chem. Res. 42, 1210-1219 (2009), https://doi.org/10.1021/ar900043h

[36]M. Ji, S. Park, and K.J. Gaffney, Dynamics of ion assembly in solution: 2DIR spectroscopy study of LiNCS in benzonitrile, J. Phys. Chem. Lett. 1, 1771-1775 (2010), https://doi.org/10.1021 iz100486x

[37] G.N. Pack, M.C. Rotondaro, P.P. Shah, A. Mandal, S. Erramilli, and L.D. Ziegler, Two-dimensional infrared spectroscopy from the gas to liquid phase: Density dependent: J-scrambling, vibrational relaxation, and the onset of liquid character, Phys. Chem. Chem. Phys. 21, 21249-21261 (2019), https://doi.org/10.1039/c9cp04101;
[38]J.D. Cyran, J.M. Nite, and A.T. Krummel, Characterizing anharmonic vibrational modes of quinones with two-dimensional infrared spectroscopy, J. Phys. Chem. B 119, 8917-8925 (2015), https://doi.org/10.1021/jp506900n

[39]Q. Guo, P. Pagano, Y.L. Li, A. Kohen, and C.M. Cheatum, Line shape analysis of two-dimensional infrared spectra, J. Chem. Phys. 142, 212427 (2015), https://doi.org/10.1063/1.4918350

[40]J. Laane, in: Frontiers of Molecular Spectroscopy (Elsevier, 2009) pp. 63-132, https://doi. org/10.1016/B978-0-444-53175-9.00004-0

[41]J. Laane, Experimental determination of vibrational potential energy surfaces and molecular structures in electronic excited states, J. Phys. Chem. A 104, 7715-7733 (2000), https://doi. org/10.1021/jp0009002

[42]J. Chen, Z. Sun, and D.H. Zhang, An accurate potential energy surface for the $\mathrm{F}+\mathrm{H}_{2} \rightarrow \mathrm{HF}+\mathrm{H}$ reaction by the coupled-cluster method, J. Chem. Phys. 142, 024303 (2015), https://doi. org/10.1063/1.4904546

[43]R. Knochenmuss, R.K. Sinha, and S. Leutwyler, Intermolecular dissociation energies of dispersively bound complexes of aromatics with noble gases and nitrogen, J. Chem. Phys. 148, 134302 (2018), https://doi.org/10.1063/1.5019432

[44]J. Zúñiga, J.A. Picón, A. Bastida, and A. Requena, A spectroscopic potential energy surface for FCN, J. Quant. Spectrosc. Radiat. Transf. 113, 1155-1169 (2012), https://doi.org/10.1016/j.jqsrt.2012.01.023

[45]W. Dong, C. Xiao, T. Wang, D. Dai, X. Yang, and D.H. Zhang, Transition-state spectroscopy of partial wave resonances in the $\mathrm{F}+\mathrm{HD}$ reaction, Science 327, 1501-1502 (2010), https://doi. org/10.1126/science.1185694

[46]Z. Yan, S.K. Gray, and N.F. Scherer, Potential energy surfaces and reaction pathways for lightmediated self-organization of metal nanoparticle clusters, Nat. Commun. 5 (2014), https://doi. org/10.1038/ncomms4751

[47]E. Skúlason, V. Tripkovic, M.E. Björketun, S. Gudmundsdóttir, G. Karlberg, J. Rossmeisl, T. Bligaard, H. Jónsson, and J.K. Nørskov, Modeling the electrochemical hydrogen oxidation 
and evolution reactions on the basis of density functional theory calculations, J. Phys. Chem. C 114, 18182-18197 (2010), https://doi. org/10.1021/jp1048887

[48]P. Pechukas, Transition state theory, Annu. Rev. Phys. Chem. 32, 159-177 (1981), https://doi. org/10.1146/annurev.pc.32.100181.001111

[49]H. Satzger, C. Root, and M. Braun, Excited-state dynamics of trans- and cis-azobenzene after UV excitation in the $\pi \pi^{*}$ band, J. Phys. Chem. A 108, 6265-6271 (2004), https://doi.org/10.1021 ip049509x

[50]P.J.M. Johnson, A. Halpin, T. Morizumi, V.I. Prokhorenko, O.P. Ernst, and R.J.D. Miller, Local vibrational coherences drive the primary photochemistry of vision, Nat. Chem. 7, 980-986 (2015), https://doi.org/10.1038/nchem.2398

[51]V. Balevičius Jr., T. Wei, D. Di Tommaso, D. Abramavicius, J. Hauer, T. Polívkae, and C.D.P. Duffy, The full dynamics of energy relaxation in large organic molecules: From photo-excitation to solvent heating, Chem. Sci. 10, 4792-4804 (2019), https://doi.org/10.1039/c9sc00410f

[52]J. Hauer, M. Maiuri, D. Viola, V. Lukes, S. Henry, A.M. Carey, R.J. Cogdell, G. Cerullo, and D. Polli, Explaining the temperature dependence of spirilloxanthin's $S^{*}$ signal by an inhomogeneous ground state model, J. Phys. Chem. A 117, 63036310 (2013), https://doi.org/10.1021/jp4011372

[53]P.G. Jambrina, L. González-Sánchez, J. Aldegunde, V. Saéz-Rábanos, and F.J. Aoiz, Competing dynamical mechanisms in inelastic collisions of H + HF, J. Phys. Chem. A 123, 9079-9088 (2019), https://doi.org/10.1021/acs.jpca.9b07272

[54]V. Sáez-Rábanos, J.E. Verdasco, and V.J. Herrero, Orbiting resonances in the $\mathrm{F}+\mathrm{HD}(v=0,1)$ reaction at very low collision energies. A quantum dynamical study, Phys. Chem. Chem. Phys. 21, 15177-15186 (2019), https://doi.org/10.1039 c9cp02718a

[55]R.J. Le Roy, Y. Huang, and C. Jary, An accurate analytic potential function for ground-state $\mathrm{N}_{2}$ from a direct-potential-fit analysis of spectroscopic data, J. Chem. Phys. 125, 164310 (2006), https://doi.org/10.1063/1.2354502
[56]E.F. de Lima and J.E.M. Hornos, Matrix elements for the Morse potential under an external field, J. Phys. B At. Mol. Opt. Phys. 38, 815-825 (2005), https://doi.org/10.1088/09534075/38/7/004

[57]J.A. Borek, F. Perakis, and P. Hamm, Testing for memory-free spectroscopic coordinates by $3 \mathrm{D}$ IR exchange spectroscopy, Proc. Natl. Acad. Sci. 111, 10462-10467 (2014), https://doi.org/10.1073 pnas.1406967111

[58]A.F. Fidler, E. Harel, and G.S. Engel, Dissecting hidden couplings using fifth-order three-dimensional electronic spectroscopy, J. Phys. Chem. Lett. 1, 2876-2880 (2010), https://doi. org/10.1021/jz101064j

[59]Z. Zhang, P.H. Lambrev, K.L. Wells, G. Garab, and H.S. Tan, Direct observation of multistep energy transfer in LHCII with fifth-order 3D electronic spectroscopy, Nat. Commun. 6, 7914 (2015), https://doi.org/10.1038/ncomms8914

[60]H. Li, A.D. Bristow, M.E. Siemens, G. Moody, and S.T. Cundiff, Unraveling quantum pathways using optical 3D Fourier-transform spectroscopy, Nat. Commun. 4, 1390 (2013), https://doi. org/10.1038/ncomms2405

[61]S. Mueller, J. Lüttig, P. Malý, L. Ji, J. Han, M. Moos, T.B. Marder, U.H.F. Bunz, A. Dreuw, C. Lambert, and T. Brixner, Rapid multiple-quantum threedimensional fluorescence spectroscopy disentangles quantum pathways, Nat. Commun. 10, 4735 (2019), https://doi.org/10.1038/s41467-01912602-x

[62]D.B. Turner and K.A. Nelson, Coherent measurements of high-order electronic correlations in quantum wells, Nature 466, 1089-1092 (2010), https://doi.org/10.1038/nature09286

[63]L. Valkunas, D. Abramavicius, and T. Mancal, Molecular Excitation Dynamics and Relaxation: Quantum Theory and Spectroscopy (Wiley-VCH Verlag GmbH \& Co. KGaA, Weinheim, Germany, 2013).

[64]Y. Tanimura, The 5th- and 7th-order 2D Raman spectroscopy for intramolecular vibrational modes, AIP Conf. Proc. 503, 144-153 (2000), https://doi.org/10.1063/1.1302858 
[65]K.J. Kubarych, C.J. Milne, and R.J.D. Miller, Fifth-order two-dimensional Raman spectroscopy: A new direct probe of the liquid state, Int. Rev. Phys. Chem. 22, 497-532 (2003), https://doi. org/10.1080/0144235031000121544

[66]B.P. Molesky, P.G. Giokas, Z. Guo, and A.M. Moran, Multidimensional resonance Raman spectroscopy by six-wave mixing in the deep UV, J. Chem. Phys. 141, 114202 (2014), https://doi.org/10.1063/1.4894846

[67]B.P. Molesky, Z. Guo, and A.M. Moran, Femtosecond stimulated Raman spectroscopy by six-wave mixing, J. Chem. Phys. 142, 212405 (2015), https://doi.org/10.1063/1.4914095

[68]J.C. Kirkwood and A.C. Albrecht, Multidimensional time-resolved coherent Raman sixwave mixing: a comparison of the direct and cascaded processes with femtosecond excitation and noisy light interferometry, J. Raman Spectrosc.
31, 107-124 (2000), https://doi.org/10.1002

(SICI)1097-4555(200001/02)31:1/2<107::AIDIRS493>3.0.CO;2-E

[69]M. Cho, D.A. Blank, J. Sung, K. Park, S. Hahn, and G.R. Fleming, Intrinsic cascading contributions to the fifth-and seventh-order electronically off-resonant Raman spectroscopies, J. Chem. Phys. 112, 2082-2094 (2000), https://doi. org/10.1063/1.480777

[70] R.D. Mehlenbacher, B. Lyons, K.C. Wilson, Y. Du, and D.W. McCamant, Theoretical analysis of anharmonic coupling and cascading Raman signals observed with femtosecond stimulated Raman spectroscopy, J. Chem. Phys. 131, 244512 (2009), https://doi.org/10.1063/1.3276684

[71]A.P. Spencer, W.O. Hutson, and E. Harel, Quantum coherence selective 2D Raman-2D electronic spectroscopy, Nat. Commun. 8, 14732 (2017), https://doi.org/10.1038/ncomms14732

\title{
NETIESINĖS SPEKTROSKOPIJOS GALIMYBĖS ATSKLEIDŽIANT INFORMACIJĄ APIE AUKŠTAI SUŽADINTAS KVANTINĖS SISTEMOS BŪSENAS
}

\author{
D. Abramavičius \\ Vilniaus universitetas, Fizikos fakultetas, Vilnius, Lietuva
}

\section{Santrauka}

Koherentine dvimate infraraudonoji ar matomosios srities spektroskopija yra labai paplitusi tyrinejjant energijos relaksacijos bei pernašos kelius daugelio pigmentų ar modų sistemose. Bendrai tai yra koreliacinė spektroskopija, kuria matuojami sąryšiai tarp skirtingų kvantinių būsenų, apsiribojant sužadinimais tik iki dviejų kvantų. Šiame straipsnyje siūloma nagrinèti daugelio kvan- tų sužadintas būsenas. Taip būtų įmanoma „skenuoti“ kvantinių sistemų netiesinius potencinius paviršius iki pat pageidaujamo sužadinimo. Tą galima pasiekti taikant paprastą trijų impulsų lazerinès spektroskopijos metodą. Jis leistų tiesiogiai gauti tikslius aukštai esančių lygmenų anharmoniškumų ịvertinimus ir šuolių tarp jų amplitudes. 